Robinson DiAs DE MEDeIROS ${ }^{1,2}$

George Dantas de Azevedo'

Técia Maria de OliverRa Maranhão ${ }^{1,2}$

ANA KATHERINE GONÇALVES ${ }^{1,2}$

YASHA EMERENCIANO BARROS ${ }^{2}$

Ana Cristina Pinhero Fernandes de Araújo ${ }^{1,2}$

STÊNIA LINS LEÃO LIMA ${ }^{3}$

Artigo Original

Palavras-chave

Saúde sexual e reprodutiva Direitos sexuais e reprodutivos Educação em saúde

Keywords

Sexual and reproductive health Sexual and reproductive rights Health education

\title{
Impacto da inserção da temática saúde sexual e reprodutiva na graduação de Medicina
}

\author{
Impact of sexual and reproductive bealth \\ theme insertion in the undergraduate medical
}

\section{Resumo}

OBJETIVO: Avaliar o impacto da inserção da temática saúde sexual e reprodutiva na graduação de Medicina em uma universidade pública do Brasil. MÉTODOS: Foi desenvolvido instrumento de avaliação cognitiva em saúde sexual e reprodutiva com base nos temas abordados no componente curricular optativo Saúde Reprodutiva, resultando em prova objetiva de múltipla escolha contendo 27 itens. Os temas selecionados foram: direitos humanos, sexuais e reprodutivos (DHSR), sexualidade, violência institucional, gênero, violência sexual, concepção, contracepção, aborto/interrupção legal da gestação, mortalidade materna e doenças sexualmente transmissíveis (DSTs) - HIV/AIDS. Os temas foram agrupados em três dimensões do conhecimento: DHSR, legal/institucional e biomédica. Na análise de covariância, dois modelos multivariados foram ajustados. RESULTADOS: Participaram do estudo 183 alunos, 127 do grupo que cursou o componente curricular eletivo saúde reprodutiva (Grupo SR) e 56 do grupo que não cursou (Grupo Não SR). Noventa e seis alunos $(52,5 \%)$ eram do sexo masculino e 87 (47,5\%) do sexo feminino. A média de idade foi de

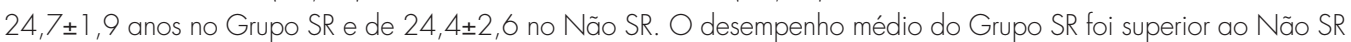
nos temas DHSR, sexualidade, violência institucional, violência sexual, aborto/interrupção legal e DSTs - HIV/AIDS. Não houve diferença no desempenho dos sexos masculino e feminino, com a exceção do tema mortalidade materna, no qual o grupo masculino foi inferior $(6,9 \pm 0,2$ e $7,8 \pm 0,2$, respectivamente; $p<0,05)$. CONCLUSÕES: A participação dos estudantes no componente curricular eletivo Saúde Reprodutiva mostrou-se associada com melhor desempenho em algumas dimensões da avaliação cognitiva, o que sugere um impacto positivo dessa iniciativa na formação médica generalista.

\section{Abstract}

PURPOSE: To evaluate the impact of sexual and reproductive health theme insertion in the undergraduate medical curriculum at a Brazilian public university. METHODS: We developed an instrument for cognitive assessment in sexual and reproductive health based on the subjects addressed in the optional curriculum component Reproductive Health, resulting in an objective multiple choice test containing 27 items. The selected topics were: human, sexual and reproductive rights (HSRR), sexuality, institutional violence, gender, sexual violence, conception, contraception, abortion/legal interruption of pregnancy, maternal mortality and sexually transmitted infections (STIS) - HIV/AIDS. The subjects were grouped into three dimensions of knowledge: HSRR, legal/institutional and biomedical. Two multivariate models were adjusted in the analysis of covariance. RESULTS: The study included 183 students, 127 of the group who took the elective curriculum course reproductive health (RH Group) and 56 who did not (Non-RH Group). Ninety-six students (52.5\%) were males and 87 (47.5\%) were females. Mean age was $24.7 \pm 1.9$ years for the RH Group and 24.4 \pm 2.6 for the Non-RH Group. The average performance of the SR Group was higher than that of Non-RH subjects regarding the following subjects: HSRR, sexuality, institutional violence, sexual violence, abortion/legal interruption, and STDs - HIV/AIDS. There was no gender difference in performance, except for the theme maternal mortality, in which males scored worse than females $(6.9 \pm 0.2$ and $7.8 \pm 0.2$, respectively; $p<0.05)$. CONCLUSIONS: The participation of students in the elective curriculum component Reproductive Health was associated with better performance in some dimensions of cognitive assessment, suggesting a positive impact of this initiative on general medical education.

Correspondência

Robinson Dias de Medeiros Departamento de Tocoginecologia da Universidade Federal do Rio Grande do Norte Avenida General Gustavo Cordeiro de Farias, $s / n$ (EP: $59012-570$ Natal (RN), Brasil

Recebido

$26 / 11 / 2013$

Aceito com modificacōes $12 / 02 / 2014$
Programa de Pós-Graduação em Ciências da Saúde da Universidade Federal do Rio Grande do Norte - UFRN - Natal (RN), Brasil. IPrograma de Pós-Graduação em Ciências da Saúde, Universidade Federal do Rio Grande do Norte - UFRN - Natal (RN), Brasil. 2Departamento de Tocoginecologia, Universidade Federal do Rio Grande do Norte - UFRN - Natal (RN), Brasil.

${ }_{3}^{3}$ Maternidade Escola Januário Cicco, Universidade Federal do Rio Grande do Norte - UFRN - Natal (RN), Brasil.

Conflito de interesses: não há 


\section{Introdução}

O direito à saúde está estreitamente vinculado a outros direitos — e depende de sua efetivação — , em particular aos direitos à vida, à alimentação, à moradia, ao trabalho, à educação, à participação, ao desfrute dos benefícios do progresso científico e suas aplicações, a estar livre de todas as formas de discriminação, à igualdade, a não ser submetido a torturas, à vida privada, ao acesso à informação e à liberdade de associação, reunião e circulação ${ }^{1}$.

A questão reprodutiva passou a fazer parte das políticas públicas, inicialmente, não como um direito geral mas como um ponto específico no âmbito das políticas de saúde ${ }^{2}$. Todavia, o próprio conceito de saúde passou por uma evolução importante nas últimas décadas. A Organização Mundial da Saúde (OMS) definiu o conceito de saúde, em 1948, da seguinte forma: “A saúde é um estado de completo bem-estar físico, mental e social e não meramente a ausência de doença ou enfermidade". Essa definição representou um avanço para a época, na medida em que definiu saúde de forma ampla e multidimensional. Mais recentemente, a OMS desenvolveu uma nova definição de saúde, que reconhece os laços entre o indivíduo e seu meio ambiente. Dessa forma, a saúde não é somente uma responsabilidade do tradicional setor saúde, mas de todos os setores e instituições que possam influenciar o bem-estar dos indivíduos e das comunidades ${ }^{3}$.

Em 1988, a OMS começou a adotar a concepção de "saúde reprodutiva". Essa inflexão foi mobilizada pela crítica aos enfoques exclusivamente biomédicos e tecnológicos que caracterizavam os programas da instituição voltados para a reprodução humana. Visava ampliar o espectro de análise, no sentido de incorporar as dimensões sociais da reprodução e desenhar políticas que não estivessem restritas ao planejamento familiar, mas pudessem responder a outras necessidades como o pré-natal, o parto, o puerpério, o aborto, as doenças ginecológicas e o câncer cervical. Essa inflexão foi um passo precursor importante no caminho que levaria em direção à Conferência Internacional sobre População e Desenvolvimento (CIPD), realizada na cidade do Cairo, em 1994, na qual, não sem razão, tal definição tornar-se-ia uma chave de leitura central dos seus resultados ${ }^{4}$.

Em 2000, a Organização das Nações Unidas (ONU), ao analisar os maiores problemas mundiais, estabeleceu os Objetivos de Desenvolvimento do Milênio (ODM), que surgiram da Declaração do Milênio das Nações Unidas, adotada pelos 191 membros dessa organização, dentre eles o Brasil. Os ODM, no que diz respeito especialmente a gênero, saúde e direitos reprodutivos, têm indicadores para as metas três e cinco. A meta três, promoção da igualdade de gênero e da autonomia das mulheres, diz respeito ao trabalho pela eliminação da violência de gênero, pela promoção da informação em saúde e em direitos sexuais e reprodutivos, por prover iguais oportunidades no mercado de trabalho e por aumentar sua participação política em todos os níveis, dentre outras questões. Com relação à meta cinco, melhorar a saúde materna, é enfatizada a prioridade de estabelecimento de um sistema de saúde funcional e bem estruturado; para que essa meta seja cumprida, deve atingir objetivos em relação ao acesso universal à anticoncepção, ao aborto seguro, à assistência pré-natal, aos sistemas de referências para assistência obstétrica de emergência e ao atendimento para as gestações de alto risco, dentre outros ${ }^{5-7}$.

Apesar dos acordos internacionais e do reconhecido avanço ocorrido no Brasil nos últimos anos com a elaboração de documentos governamentais e a implantação do Sistema Único de Saúde (SUS), as brasileiras continuam sofrendo discriminações e violações e enfrentando barreiras no acesso à saúde, sendo vítimas de processos de exclusão em vários níveis e esferas ${ }^{8}$. A carência de informação, bem como as precárias políticas de saúde são refletidas nos elevados índices de mortalidade materna, infecção por DSTs e HIV, gestações indesejadas, abortos inseguros e violência doméstica e sexual ${ }^{9}$. Por outro lado, a incorporação de temas relacionados à saúde sexual e reprodutiva permanece deficiente na formação profissional em saúde ${ }^{10}$.

Considerando a importância do tema, especificamente para a saúde das mulheres, a proposta do presente estudo foi avaliar o impacto da inserção da temática saúde sexual e reprodutiva na graduação de Medicina em uma universidade pública do Nordeste brasileiro.

\section{Métodos}

Este foi um estudo transversal que incluiu 183 alunos do curso de Medicina da Universidade Federal do Rio Grande do Norte (UFRN) que estavam cursando o internato $\left(9^{\circ}\right.$ ao $12^{\circ}$ períodos) durante o ano de 2011. Foi desenvolvido um instrumento de avaliação cognitiva em saúde sexual e reprodutiva contendo 27 questões objetivas, com base nos temas sistematicamente abordados no componente curricular optativo Saúde Reprodutiva, ofertado a partir do ano de 2006 para alunos do $5^{\circ}$ ao $8^{\circ}$ períodos do curso. Os temas selecionados foram: direitos humanos, sexuais e reprodutivos (DHSR), sexualidade, violência institucional, gênero, violência sexual, concepção, contracepção, aborto/interrupção legal da gestação, mortalidade materna e DSTs - HIV/AIDS. Os temas foram agregados em três dimensões de conhecimento consideradas: DHSR, legal/institucional e biomédica.

O protocolo de pesquisa foi avaliado e aprovado pelo Comitê de Ética em Pesquisa do Hospital Universitário Onofre Lopes (CEP - HUOL), sob o número 479/10.

Os dados gerados pela pesquisa foram codificados e registrados em planilha do Microsoft Office Excel $2003^{\circledR}$. 
Em seguida, foi construído Banco de Dados (BD) associado a programas de análise estatística como o PASW Statistics Grad Pack $18^{\circledR}$ (www.spss.com) ou Statistica 6.1 ${ }^{\circledR}$ (www.statsoft.com). Foram construídas variáveis derivadas dos registros originais, sobre as quais foram aplicadas ferramentas de análise. Os itens da avaliação cognitiva resultaram em 27 questões dicotômicas (certo/errado). Além desses resultados, foram registrados o sexo e a idade do estudante e se ele havia cursado ou não o componente curricular optativo Saúde Reprodutiva (Grupos SR e Não $\mathrm{SR}$, respectivamente). Foram definidos 10 temas em função de subconjuntos das 27 questões da prova objetiva, denotando Erro=0 ou Acerto=1. O desempenho em cada tema foi avaliado por 10 vezes a média das questões Qi a ele pertencentes, gerando uma nota no intervalo 0-10. Após a definição dos dez temas, foram definidas três dimensões como sendo a média das notas dos temas a elas pertencentes. A hipótese nula multivariada sob teste é a de que "os fatores Grupo (SR e Não SR), sexo (masculino e feminino) e a covariável idade não têm efeito sobre o perfil médio de resposta" formado pelos dez temas, bem como sobre o perfil médio de respostas formado pelas três dimensões. Para avaliar tal hipótese, foram ajustados dois modelos multivariados de covariância (ANCOVA).

Os resultados também foram ilustrados por meio de gráficos de perfil médio com intervalos de confiança de $95 \%$. Média, desvio padrão (DP) e intervalo de confiança de $95 \%$ (IC95\%) são apresentados em tabelas para temas e dimensões por grupo e sexo.

\section{Resultados}

Fizeram parte do estudo 183 alunos do internato do curso de Medicina da UFRN, dos quais 127 eram do grupo que cursou o componente curricular eletivo Saúde Reprodutiva (Grupo SR) e 56 do grupo que não cursou o componente curricular eletivo Saúde Reprodutiva (Grupo Não SR). Noventa e seis alunos $(52,5 \%)$ eram do sexo masculino e $87(47,5 \%)$ eram do sexo feminino. A idade

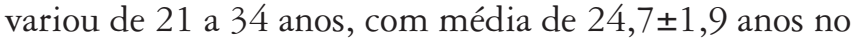

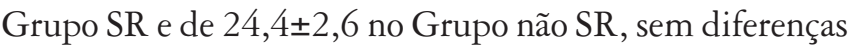
estatisticamente significativas.

A Tabela 1 apresenta estimativas amostrais da média, DP e IC95\% para o desempenho dos estudantes na prova cognitiva de acordo com a participação ou não no componente eletivo Saúde Reprodutiva e o sexo. Foi observado que o desempenho médio do Grupo SR foi significativamente superior ao do Grupo Não SR nos temas DHSR, sexualidade, violência institucional, violência sexual, aborto/interrupção legal e DSTs - HIV/AIDS. Também foi notado que ocorreu uma homogeneidade nas performances dos estudantes nos diversos temas, exceto no que tange ao desempenho relacionado ao tema concepção, no qual ocorreu um menor desempenho global (3,2 $\pm 0,4$ e 2,6 $\pm 0,6$ nos Grupos SR e Não SR, respectivamente; $p>0,05)$.

Adicionalmente, a análise univariada do efeito da participação no componente optativo Saúde Reprodutiva, sexo e idade sobre o desempenho dos estudantes na avaliação cognitiva reforçou os dados apresentados na Tabela 1 ,

Tabela 1. Desempenhos médios dos estudantes na avaliação cognitiva de acordo com as temáticas abordadas e os Grupos Saúde Reprodutiva e Não Saúde Reprodutiva relacionadas ao sexo

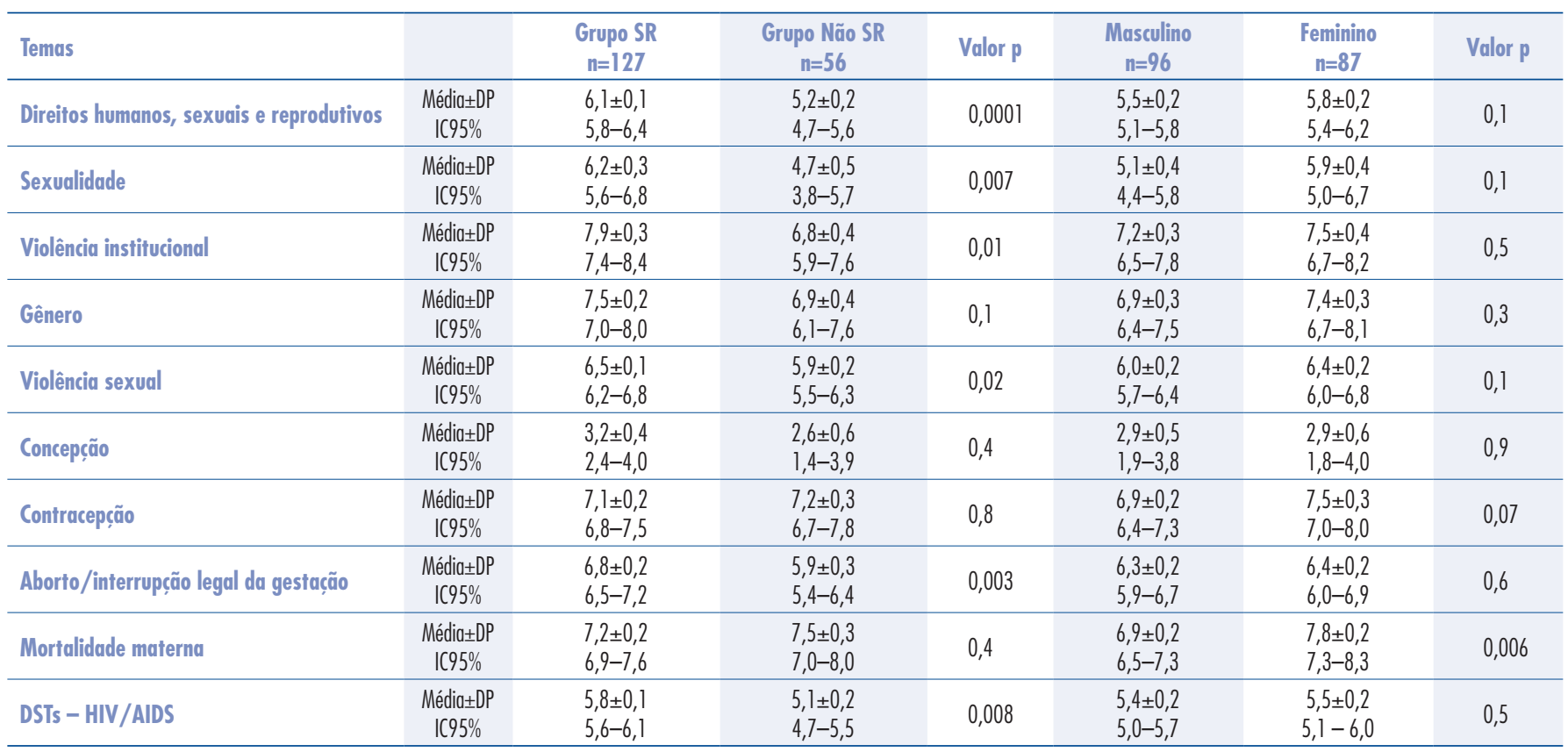

Grupo SR: estudantes que cursaram o componente curricular optativo Saúde Reprodutiva; Grupo Não SR: estudantes que não cursaram o componente curricular citado. 
tendo em vista que no Grupo SR o parâmetro estimado beta foi positivo em seis dentre os dez temas elencados, com significância estatística resultando em um incremento médio das notas dos estudantes em relação aos colegas do Grupo Não SR.

Ainda na Tabela 1 é possível observar que não houve diferença estatisticamente significativa entre os desempenhos de estudantes dos sexos masculino e feminino para a maioria dos temas analisados, com a exceção do tema mortalidade materna, no qual o desempenho do grupo masculino foi expressivamente inferior ao do grupo feminino $(6,9 \pm 0,2$ e $7,8 \pm 0,2$, respectivamente; $\mathrm{p}<0,05)$. O modelo linear ajustado reafirma esse resultado, demonstrando que existe efeito significativo do sexo sobre o desempenho dos estudantes somente em relação ao tema mortalidade materna $(\beta=-0,44 ; p=0,006)$.

A Tabela 2 apresenta os desempenhos médios dos estudantes pertencentes aos Grupos SR e Não SR em relação a três dimensões de conhecimento, definidas a partir da agregação dos temas abordados nos itens constantes da prova cognitiva, a saber: dimensão direitos humanos, sexuais e reprodutivos (DHSR), dimensão legal/institucional e dimensão biomédica. Foi percebido que o Grupo SR teve desempenho consideravelmente superior ao Grupo Não SR nas dimensões DHSR e legal/institucional.

Após a análise dos resultados do modelo linear ajustado e tendo como resposta as três dimensões de conhecimento consideradas, é notado efeito significativamente positivo do Grupo SR nas dimensões DHSR e legal/institucional, demonstrando que os estudantes que cursaram previamente o componente Saúde Reprodutiva obtiveram um desempenho superior aos que não cursaram em relação às duas dimensões citadas. Adicionalmente, é percebido que existe interação significativa e positiva entre os fatores Grupo SR e sexo masculino nas mesmas dimensões DHSR e legal/institucional. Isso significa dizer que o efeito do Grupo SR depende do sexo, uma vez que ele é ainda maior no sexo masculino, com um diferencial $\beta=0,27$ na dimensão DHSR e um diferencial $\beta=0,26$ na legal/institucional.

Tabela 2. Desempenhos médios dos estudantes na avaliação cognitiva, de acordo com as dimensões de conhecimento consideradas e os grupos Saúde Reprodutiva e Não Saúde Reprodutiva

\begin{tabular}{|c|c|c|c|c|}
\hline Dimensões & & $\begin{array}{c}\text { Grupo SR } \\
n=127\end{array}$ & $\begin{array}{c}\text { Grupo não SR } \\
n=56\end{array}$ & Valor $p$ \\
\hline $\begin{array}{l}\text { Direitos humanos, } \\
\text { sexuais e reprodutivos }\end{array}$ & $\begin{array}{l}\text { Média } \pm \text { DP } \\
\text { IC95\% }\end{array}$ & $\begin{array}{l}6,0 \pm 0,1 \\
5,7-6,3\end{array}$ & $\begin{array}{l}5,1 \pm 0,2 \\
4,6-5,5\end{array}$ & 0,001 \\
\hline Legal/institucional & $\begin{array}{l}\text { Média } \pm D P \\
\text { IC95\% }\end{array}$ & $\begin{array}{l}6,4 \pm 0,1 \\
6,2-6,7\end{array}$ & $\begin{array}{l}6,0 \pm 0,2 \\
5,6-6,4\end{array}$ & 0,04 \\
\hline Biomédica & $\begin{array}{l}\text { Média } \pm D P \\
\text { IC95\% }\end{array}$ & $\begin{array}{l}5,8 \pm 0,1 \\
5,6-6,1\end{array}$ & $\begin{array}{l}5,6 \pm 0,2 \\
5,2-6,0\end{array}$ & 0,3 \\
\hline
\end{tabular}

Grupo SR: estudantes que cursaram o componente curricular optativo Saúde Reprodutiva; Grupo Não SR: estudantes que não cursaram o componente curricular citado.

\section{Discussão}

A implementação de uma nova estrutura curricular no curso de Medicina da UFRN, em 2001, deu sequência à incorporação de novos cenários e métodos de ensino-aprendizagem, bem como às experiências inovadoras de integração de saberes disciplinares. Sob essa nova perspectiva, a escola passa a ser um espaço onde a cidadania deverá ser desenvolvida, e o professor tem grande responsabilidade nesse desenvolvimento, na medida em que reconhece o aluno como um futuro cidadão conhecedor dos direitos e deveres perante a sociedade em que atuará ${ }^{11}$.

A disciplina de Saúde Reprodutiva, ofertada semestralmente a partir de 2006, é parte integrante desse processo e, junto com outros componentes curriculares, passou a ter importância na formação e avaliação das diferentes habilidades envolvidas na atenção à saúde sexual e reprodutiva das pessoas. Reconhecer a necessidade desses conhecimentos e habilidades, os pontos fortes, as fragilidades e as lacunas existentes remete a uma avaliação desse processo, ao mesmo tempo em que motiva o envolvimento com a formação médica. Neste trabalho, são apresentados os resultados obtidos com a participação dos graduandos do internato de Medicina durante o ano de 2011, como reflexo de diversas iniciativas de reorientação curricular, especificamente no que tange à incorporação das temáticas relacionadas à saúde sexual e reprodutiva.

Os resultados observados na avaliação das habilidades cognitivas demonstraram que os alunos que cursaram o componente curricular Saúde Reprodutiva tiveram desempenho significativamente superior em temas como DHSR, sexualidade, violência institucional, violência sexual, aborto/interrupção legal e DSTs - HIV. De certa forma, essa era a hipótese esperada na pesquisa, tendo em vista a suposição de que, ao cursar a disciplina Saúde Reprodutiva, os alunos teriam mais oportunidades de obter conhecimentos e incorporar conceitos sobre questões significativas na atenção à saúde.

O resultado destacado é um dado relevante para a análise da pesquisa e das questões relacionadas à atenção de qualidade. Um estudo que aborda a profissão médica e a defesa e promoção dos direitos sexuais e reprodutivos chama a atenção para o fato de que os profissionais de saúde, por terem status, perícia, credibilidade e contatos com a comunidade em geral, estão em uma posição excepcional na sociedade para a garantia do direito à saúde, tanto no âmbito de sua atuação profissional como no âmbito de sua participação política, e para o fato de que essas prerrogativas são importantes na elaboração de políticas em prol da mudança e da melhoria da atenção na área de saúde sexual e reprodutiva ${ }^{12}$.

$\mathrm{Na}$ análise do desempenho de acordo com o sexo e os diversos temas da avaliação de conhecimentos, um achado 
interessante foi a observação de que o grupo feminino apresentou desempenho significativamente superior ao grupo masculino no tema mortalidade materna. Esse dado aponta para a importância da consideração da questão de gênero na formação profissional em saúde, conforme já previamente demonstrado em estudos que destacaram, por exemplo, diferenças relacionadas ao gênero nas atitudes de estudantes de Medicina no cuidado aos pacientes ${ }^{13,14}$. Em estudo semelhante realizado em uma universidade do Sudeste brasileiro, foram avaliadas as atitudes de estudantes de diferentes níveis do curso no que diz respeito à relação médico-paciente, utilizando o questionário Patient-Practitioner Orientation Scale (PPOS). Os resultados demonstraram que o gênero feminino foi expressivamente associado com atitudes mais centradas no paciente, e foi sugerida pelos autores a necessidade das escolas médicas examinarem criticamente esses aspectos na formação dos estudantes ${ }^{13}$.

Sobre a temática mortalidade materna, esse é um conteúdo rotineiramente abordado no curso de Medicina da UFRN, não só no componente curricular Saúde Reprodutiva mas também em outros componentes curriculares obrigatórios. Este é um problema de saúde pública $^{15}$, dependente de questões culturais e educacionais e de políticas públicas de saúde ${ }^{16}$. Como tal, é plausível admitir que sua adequada abordagem, especialmente no processo formativo, sofra influência de diversos fatores de ordem pessoal e subjetiva, incluindo-se aí a sensibilidade social e as qualidades humanísticas.

Por outro lado, a relação homem e saúde é objeto de atenção nos meios acadêmicos e também no contexto dos serviços, ganhando relevância nas últimas décadas, especialmente nas análises da sexualidade e da saúde reprodutiva $^{17,18}$. Os trabalhos têm abordado os homens por meio de distintas perspectivas: de forma instrumental, como apoio à saúde das mulheres; pelo reconhecimento de suas necessidades de informação ou de saúde; pela responsabilização por práticas sexuais de risco; e de afirmação da necessidade de sua participação nas questões de saúde reprodutiva e sexual como integrante dos direitos reprodutivos ${ }^{19-21}$. O resultado encontrado neste estudo é surpreendente e interessante por suscitar a discussão a respeito da influência das questões de gênero, masculinidade e relação com o cuidado em saúde sobre o processo de aprendizagem.

$\mathrm{Na}$ atualidade, o médico egresso necessita de competências, habilidades e atitudes que atendam às exigências e às necessidades da sociedade contemporânea. Dessa forma, cada vez mais, esse profissional vem sendo chamado a pensar sobre problemas coletivos, ou seja, que não estão no âmbito da saúde de um único indivíduo. Questões abrangentes como direito à saúde, acesso aos serviços, autonomia reprodutiva e redução de danos merecem esforços coletivos para promover o desenvolvimento de outras competências, quais sejam: políticas, éticas e humanas. Estas vão se agregando e constituindo uma nova visão no âmbito da formação, da atuação e do planejamento em saúde ${ }^{22-24}$.

De acordo com essa ótica e por meio da análise dos resultados da avaliação das três dimensões englobadas na avaliação cognitiva, mereceu destaque o desempenho superior e consideravelmente positivo dos alunos que cursaram a disciplina Saúde Reprodutiva nas dimensões DHSR e legal/institucional, o que esteve de acordo com a proposta da disciplina de fortalecer a formação acadêmica dos alunos não somente nos componentes teórico-metodológicos e técnico-operativos mas também nos componentes ético-políticos ${ }^{18,24-26}$.

Cabe aqui destacar a relevância do estudo e da adequação curricular, tendo em vista o ineditismo da iniciativa na comunidade científica brasileira e no âmbito da educação médica. Face à fragilidade evidenciada nas práticas de atenção à saúde, é salientada a necessidade de estudos adicionais direcionados para a avaliação de habilidades clínicas e de atitudes dos estudantes ante aos reais problemas relacionados à saúde sexual e reprodutiva das pessoas.

\section{Agradecimentos}

A Maria do Carmo Lopes de Melo (professora adjunta do Departamento de Tocoginecologia da UFRN), pelas sugestões e pela colaboração com o desenvolvimento do presente estudo; e ao professor José Wilton Queiroz, pela consultoria e colaboração na análise estatística.

\section{Referências}

1. Mclntosh CA, Finkle JL. The Cairo Conference on Population and Development: a new paradigm? Popul Dev Rev. 1995;21(2): 223-60

2. Braveman $P$, Gruskin S. Poverty, equity, human rights and health. Bull World Health Organ. 2003;81 (7): 539-45.

3. World Health Organization. A conceptual framework for action on the social determinants of health. Geneva: WHO; 2010.
4. AbouZahr C. Safe Motherhood: a brief history of the global movement 1947-2002. Geneva: WHO; 2002.

5. Brasil. Presidência da República. Objetivos de desenvolvimento do milênio: relatório nacional de acompanhamento. Brasília (DF): IPEA; 2010.

6. Chirawatkul S. Women's health and the Millennium Development Goals: a long way to go. Nurs Health Sci. 2012;14(3):275-6. 
7. World Health Organization. Millennium Development Goals. New Delhi: United Nations/WHO; 2008.

8. Galvão L. Saúde sexual e reprodutiva no Brasil. São Paulo: Hucitec; 1999.

9. World Health Organization [Internet]. Unsafe abortion: global and regional estimates of the incidence of unsafe abortion and associated mortality in 2000. 4th ed. Geneva: WHO; 2004 [cited 2009 Oct 10]. Available from: http://whqlibdoc. who.int/ publications/2004/9241591803.pdf?ua=1.

10. Goldstein I. Integrating women's sexual health into women's health education. J Sex Med. 2012;9(12):2983-5.

11. Bydlowski CR, Levèvre AM, Pereira IMTB. Promoção da saúde e a formação cidadã: a percepção do professor sobre cidadania. Ciênc Saúde Coletiva. 201 1;16(3):1771-80.

12. Briozzo L, Faúndes $A$. The medical profession and the defense and promotion of sexual and reproductive rights. Int J Gynaecol Obstet. 2008; 100(3):291-4.

13. Ribeiro MM, Krupat E, Amaral CF. Brazilian medical students' attitudes towards patient-centered care. Med Teach. 2007;29(6):e204-8.

14. Wahlqvist M, Gunnarsson RK, Dahlgren G, Nordgren S. Patient-centred attitudes among medical students: gender and work experience in health care make a difference. Med Teach. 2010;32(4):e191-8.

15. Rede Interagencial de Informações para a Saúde. Indicadores básicos para a saúde no Brasil: conceitos e aplicações. $2 a$ ed. Brasília (DF): Organização Pan-Americana da Saúde; 2008.

16. Souza JP. [Maternal mortality in Brazil: the need for strengthening health systems]. Rev Bras Ginecol Obstet. $2011 ; 33(10): 10-13$. Portuguese.
17. Machin R, Couto MT, Silva GSN, Schraiber LB, Gomes R, Figueiredo WS, et al. Concepções de gênero, masculinidade e cuidados em saúde: estudo com profissionais de saúde da atenção primária. Ciênc Saúde Coletiva. 2011 ; 16(11):4503-12.

18. Shindel AW, Parish SJ. Sexuality education in North American medical schools: current status and future directions. J Sex Med. 2013;10(1):3-17.

19. Giffin K. A inserção dos homens nos estudos de gênero: contribuições de um sujeito histórico. Ciênc Saúde Coletiva. $2005 ; 10(1): 47-57$.

20. Schraiber LB, Gomes RG, Couto MT. Homens e saúde na pauta da saúde coletiva. Ciênc Saúde Coletiva. 2005; 10(1): 7-17.

21. Medrado B, Lyra J. Por uma matriz feminista de gênero para os estudos sobre homens e masculinidades. Rev Estud Fem. 2008; 16(3):809-40.

22. Cook RJ, Dickens BM, Fathalla MF. Reproductive health and human rights: integrating medicine, ethics, and law. Oxford: Clarendon; 2003.

23. Norcini J, Anderson B, Bollela V, Burch V, Costa M, Duvivier R, et al. Criteria for good assessment: consensus statement and recommendations from the Ottawa 2010 Conference. Med Teach. $2011 ; 33(3): 206-14$.

24. Leaning J. Humans rights and medical education. BM. 1997;315(7120):1390-1.

25. Cook RJ, Dickens BM, Fathalla MF. Saúde reprodutiva e direitos humanos: integrando Medicina, ética e direito. Rio de Janeiro: CEPIA/Oxford; 2004

26. Medeiros RD, Azevedo GD, Ferro D, Costa RC. Human, sexual and reproductive rights in health care. Med Educ. $2011 ; 45(11): 1139$. 